\title{
PAIN MANAGEMENT KNOWLEDGE OF REGISTERED NURSES PRACTICING IN ACUTE-CARE HOSPITALS IN THE BAHAMAS
}

By Shane Neely-Smith, Maggie Turner, Zorene A. Curry, Theresa E. Moxey-Adderley, Constance J. Wilson \& Sandra G. Smith https://doi.org/10.15362/ijbs.v12i0.51

\section{Abstract}

Since pain is the most common complaint of the hospitalized patient and nurses are with patients more than any other healthcare professionals, it is imperative that nurses know how to adequately assess and manage patients' pain. However, findings from studies have suggested that nurses' lack of knowledge is among the primary reasons for inadequate pain management, which leads to major discomfort and decreased quality of life for patients. A descriptive, correlational study was conducted to describe and explore the knowledge base of registered nurses practicing in acute-care hospitals in The Bahamas in regard to pain management. The "model of nursing knowledge," developed by Jacobs-Kramer and Chinn (1988), was used as the conceptual framework for this study. The population was 247 registered nurses from three hospitals that offer acute care in The Bahamas. Data were collected via a questionnaire, which consisted of three sections: (a) six demographic items, (b) 15 multiple-choice items, and (c) 15 true or false items. Sections B and C contained items originally designed by McCaffery and Ferrell (1992), some of which were modified to reflect the pain management practices in The Bahamas. The mean pain management knowledge score was $13.5(46.6 \%$, se $=0.20)$. Findings indicated that nurses lack pain management knowledge concerning physical and behavioral indicators of pain, pain assessment principles, identification of opioids/narcotics, equianalgesia, and that they believe in many myths related to pain assessment and management. The results from this study reveal the need to develop strategies that will enhance pain management practices for the hospitalized patient to increase quality of life and patient satisfaction. 


\section{Introduction}

Pain is considered a private, personal sensation of hurt manifested by sensory and behavior components and influenced by physiological, psychological, and sociological factors (Ignatavicius, Workman, \& Mishler, 1999). It is the most common human complaint with a high probability to affect the quality of life, and yet is very problematic in its management, due to its high degree of subjectivity and health professionals' lack of knowledge regarding its management. According to McCaffery, "Pain is whatever the experiencing person says it is and exists whenever he says it does" (Ignatavicius et al., 1999, p. 109). It is this personal experience of pain that historically, and still today, is partly responsible for its ineffective management. Good pain management is dependent on good pain assessment, which includes the knowledge and ability of the assessor. Since nurses spend more time with patients than any other health professional, they witness patients' experience with pain, and, therefore, have a pivotal role to play in pain assessment and management (White, 1999).

According to C. Richard Chapman, president of the American Pain Society, of the 23 million surgical operations performed in the United States yearly, only one in four persons receives adequate pain relief. Additionally, four in ten persons out of 50 million persons who suffer with chronic pain do not receive adequate relief of pain, and of the numerous cancer victims in the country, only about $30 \%$ are adequately relieved of their pain (Phillips, 2000).

Despite efforts to improve pain management, many studies have shown that nurses are not assessing pain accurately. The main barriers to adequate pain management are nurses' lack of knowledge concerning the types of pain and pain medications, including effects and side effects, and their belief system regarding pain (Bowman, 1994; Camp \& O'Sullivan, 1987; Dalton, 1989; Dufault, Bielecki, Collins \& Willey, 1995; Gujol, 1994; Harrison, 1991; Herr \& Mobily, 1993; Rutledge \& Donaldson, 1998a; Rutledge \& Donaldson, 1998b; Simons \& Malabar, 1995; Wells, 1984; Willson, 2000). Consequently, these barriers result in under medication or over medication of patients' pain and a decrease in their quality of life.

\section{Problem statement}

Since nurses spend more time with patients than any other healthcare professionals, they play an essential role in managing patients' pain. Unfortunately, many patients experience decreased quality of life because nurses lack knowledge regarding adequate pain assessment and management. Since the pain management practices of nurses have not been addressed in The Bahamas, the purpose of this study was to describe and explore the knowledge level of registered nurses practicing in acute-care hospitals in The Bahamas in regard to pain management. The following questions were addressed:

1. What is the pain management knowledge of registered nurses practicing in acute-care hospitals in The Bahamas?

2. Is there a difference in pain management knowledge level of registered nurses practicing in acute-care hospitals in The Bahamas among hospitals?

3. What is the relationship between registered nurses practicing in acutecare hospitals in The Bahamas highest educational level and their 
knowledge of pain management?

4. What is the relationship between registered nurses practicing in acutecare hospitals in The Bahamas years of clinical experience and their knowledge of pain management?

\section{Literature Review}

There are countless studies in the literature about nurses' pain management practices. However, findings from these studies revealed that nurses inadequately assess and manage patients' pain.

Kubecka, Simon, and Boettcher (1996) conducted a study with 123 registered nurses to determine the pain management knowledge of hospital-based nurses in a rural Appalachian area. While findings showed adequate knowledge in some areas of pain management, they found that nurses lacked knowledge about the behavioral indicators of pain, classification of opioids and non-opioids, and incidence of addiction due to opioids. Similarly, a survey conducted by McCaffery, Ferrell, O’Neil-Page and Lester (1990) during a series of pain management workshops throughout the United States revealed that nurses lack knowledge concerning opioids, other medications used to treat pain, and frequency of addiction from opioids. Incidentally, however, there were no differences in the pain management knowledge between expert nurses and novice nurses. Four years later, Gujol (1994) conducted a study using a convenience sample of 71 critical care nurses to explore the relationship between pain assessment and medication decision and to identify nurses' concerns about opioid use. Interestingly, the results suggested that nurses still lack knowledge in pain assessment and management, indicating inadequate pain management and, thus, decreased quality of life for the patients.

According to Ferrell, Eberts, McCaffery and Grant (1991), a survey conducted to determine nurses' clinical decision making in relation to assessment and relief of pain found that nurses identified lack of knowledge as one of the barriers to providing optimum pain relief for their patients. In addition, Rutledge and Donaldson (1998a; 1998b) evaluated a composite of research studies, literature reviews, and conference presentations on pain management and concluded that health professionals, including nurses, generally lacked knowledge about pain assessment and management.

For nurses to assess pain accurately and provide adequate pain management for their patients, it is incumbent upon them to be knowledgeable about the physiology of pain, as well as factors that influence pain management, such as age, gender, and culture. Moreover, nurses should be knowledgeable about the different pain theories and signs of acute and chronic pain to accurately assess pain in the patient who is unable to self-report pain (Ignatavicius et al, 1999).

Due to advancements in technology, people are living longer. Unfortunately, increased quality of life is not promised with increased age, leaving the elderly vulnerable to pain. Moreover, the elderly experience more sensory defects, for example, decreases in hearing and visual acuity, psychomotor, and cognitive alterations, than any other developmental group (Herr \& Mobily, 1993). In a pilot study of nurses assessing pain in elderly patients who could not respond verbally, Simons and Malabar (1995) found that 39 out of 82 hospitalized 
patients studied were unable to communicate their pain. The study required nurses to record the patients' pain behaviors, pain interventions given, and pain reassessments using an observable pain assessment chart. The results suggested that pain interventions changed the behaviors of the majority of patients, which implied decreased pain or pain relief. This study suggests that due to nurses' adequate knowledge concerning pain assessment, adequate pain management was employed.

According to Chambers, Cassidy, McGrath, Gilbert and Craig (1996), self-report of pain is not possible in children younger than three years old and children who are developmentally delayed. Therefore, it is imperative for health professionals to assess pain in this age group using observable indicators. Nurses have to be knowledgeable in behavioral and physiological signs of pain in this age group in order to accurately assess and adequately manage their pain.

Calvillo and Flaskerud (1993) conducted a study to examine the relationship between pain and ethnicity with Mexican-American women and AngloAmerican women post-surgery, while hospitalized. They concluded that there were no significant differences in pain scores between these two groups. However, they found significant differences between nurses' evaluation of their patients' pain and patients' evaluation of their own pain. Also, nurses rated pain higher in the Anglo-American patients than in their MexicanAmerican counterparts. Therefore, nurses need adequate knowledge in pain management in order to detect and prevent personal biases.

In a study conducted by McDonald, McNulty, Erickson and Weiskopf (2000), discharged Latino, Anglo, and African-American patients were interviewed about their post-operative pain management while in hospital. The sample consisted of 30 patients, half of whom were women, placed in groups of ten. Although these patients reported various intensities of pain while hospitalized, there were not many gender and cultural differences found among them. Interestingly enough, three African-American men stated that they wanted pain medication while in the hospital but did not ask for it, and later wished they had. Still, they did not know why they did not ask for their pain medication. It would be interesting to find out what further studies might reveal with a larger and more diverse sample size, in relation to how culture and gender might influence the type of pain management patients receive. Nevertheless, nurses need to be aware of cultural and gender differences to manage pain adequately. In terms of nurses' beliefs concerning gender and pain, McCaffery and Ferrell (1992) found that nurses expected the patient's gender to influence their pain responses. Additionally, nurses exhibited numerous gender biases, which may result in their inaccurate assessment of pain and inadequate pain management. This is in keeping with a study conducted by Yates et al. (1998) on the prevalence and perception of pain amongst hospital in-patients. Besides the fact that the study showed that pain affected patients' well-being in terms of mobility and sleep, it also found that women were less willing to report their pain than men. This finding suggests that women need to be encouraged to self-report pain for adequate management. However, nurses can only educate patients, their families and/or their significant others about pain management and encourage them to report pain if they themselves are well versed on the topic. 
The American Pain Association (APS) and Agency for Health Care Policy and Research (AHCPR) state that the patient is in authority of his or her pain management, and self-report is paramount for accurate pain assessment and adequate management (Ben David \& Musgrave, 1996; Bowman, 1994; Calvillo \& Flaskerud, 1993; Camp \& O’Sullivan, 1987; Dalton, 1989; Dufault et al., 1995; Gujol, 1994; Herr \& Mobily, 1993; Ignatavicius et al., 1999; Nash, Edwards \& Nebauer, 1992; Rutledge \& Donaldson, 1998a, Rutledge \& Donaldson, 1998b; White, 1999). In an attempt to objectify the patient's subjective experience of pain, many types of pain-rating tools were developed. As a result, nurses need to be knowledgeable about the use of these tools for adequate pain management.

Bowman (1994) conducted a descriptive study and compared 13 nurses' and 16 patients' perceptions of surgical pain. Using a pain-rating scale, patients were asked to rate their pain and nurses were asked to record their rating of the patient's pain after assessment. Results suggested significant differences in the nurses' perception and the patients' perception of pain as indicated on the rating scale. Moreover, nurses underrated the patient's pain most of the time. The results of this study are evidence that patients' self-report and the use of a pain-rating scale are beneficial in adequate pain management, and, therefore, that nurses should be knowledgeable about pain-rating scales.

\section{Conceptual Framework}

Jacobs-Kramer and Chin (1988) developed a "Model of nursing knowledge" based on the foundation of Carper's (1978) "Fundamental patterns of knowing in nursing." This knowledge base includes "empirical" knowledge of facts and theories; "ethical" knowledge of standards, codes, and theories; "personal" knowledge of the authentic self and nurse/patient relationship; and "esthetic" knowledge of art, creativity, and action. According to this model, nurses should base their practice on all of these patterns of knowing to enhance its effectiveness.

The participants' empirical knowledge base was tested through scientific items on the questionnaire related to pain management. Additionally, their ethical, esthetic, and personal knowledge bases were also tested through items on the questionnaire designed to determine their decision-making and actions related to pain management practices in acute-care hospitals in The Bahamas.

\section{Methodology}

A cross-sectional descriptive survey design was used to study the knowledge level of registered nurses practicing in acute-care hospitals in The Bahamas in regard to pain management. Registered nurses were asked to complete a questionnaire to obtain demographic and pain management information.

Population. The target group for this study was all registered nurses (approximately 500), less 50 who participated in the pilot study, practicing in acutecare hospitals in The Bahamas.

Setting. Three acute-care hospitals situated on two of the central islands of The Bahamas were used for the study: Hospital A, a 450-bed, non-profit, public hospital; Hospital B, a 108-bed, public hospital, and Hospital C, a 72-bed private hospital. 
Instrumentation. The instrument was a pain management survey consisting of three sections (see Appendix A). The first section consisted of demographic questions including age, gender, years of experience as a registered nurse, current clinical area, length of time in current clinical area, and highest degree earned in nursing.

The second section consisted of 15 multiple-choice questions and the third section consisted of 15 true/false questions. These two sections were designed by McCaffery in 1988 and updated in 1990 and 1992. The sections include questions regarding pain assessment and management, including pharmacological and non-pharmacological interventions. However, the sections were modified and included four additional questions taken from the literature review to reflect pain management practices in The Bahamas.

More than 3,500 nurses in the United States and Canada have used McCaffery's pain management tools (Kubecka et al., 1996). The content was extracted from established pain management guidelines of the Agency for Health Care Policy and Research (AHCPR), World Health Organization (WHO), and the American Pain Society (APS). Additionally, participants' scores in the pilot, post pilot, and main studies showed significant correlation, $r=0.619 ; r=0.899$; and $r=$ 0.734, respectively, suggesting reliability of the instrument. Additionally, the questionnaires were color-coded to identify each participating hospital.

\section{Data collection procedure and Ethical Considerations.}

After obtaining permission from the appropriate hospitals' authorities, questionnaires accompanied by a cover letter ensuring anonymity were hand delivered by the researcher and four assistants to on-duty, registered nurses in all three hospitals over a period of three days, all shifts, simultaneously.

Participants were given a simple overview of the proposed study, and, after consenting to participate, were encouraged to complete the questionnaire as accurately as possible, which took approximately 20 minutes. The investigators either waited for the participants to complete the questionnaires or returned for the questionnaires before the end of the shift.

\section{Results}

Two hundred and ninety-four questionnaires were hand delivered and 247 were returned for a response rate of $84 \%$. However, only 244 questionnaires were used for the analyses, mainly because of too many missing responses to the questions. Sixty-six percent of the participants were from Hospital A ( $n=$ $154) ; 16 \%$ from Hospital B $(n=37)$; and $18 \%$ from Hospital C $(n=42)$ (Table 1$)$. Ninety-three percent of the participants were females $(n=218)$ and $7 \%$ were males $(n=16)$. Most of the participants were between the ages of 31 and 40 years $(43 \%)$. Their average nursing experience was 12.3 years, with a range of less than 1 year to 42 years. Their average time spent in their current clinical area was 4 years. The largest number of participants practiced in the surgical (29\%, $n=60)$ and medical $(21 \%, n=43)$ areas. Almost half $(48 \%, n=121)$ of the participants had a diploma as their highest degree earned in nursing. Thirtythree percent had an associate degree $(n=81), 9 \%$ had a baccalaureate degree $(n=21)$, and $3 \%$ had a master's degree $(n=9)$ (Table 2$)$. 


\section{Sample by Hospital}

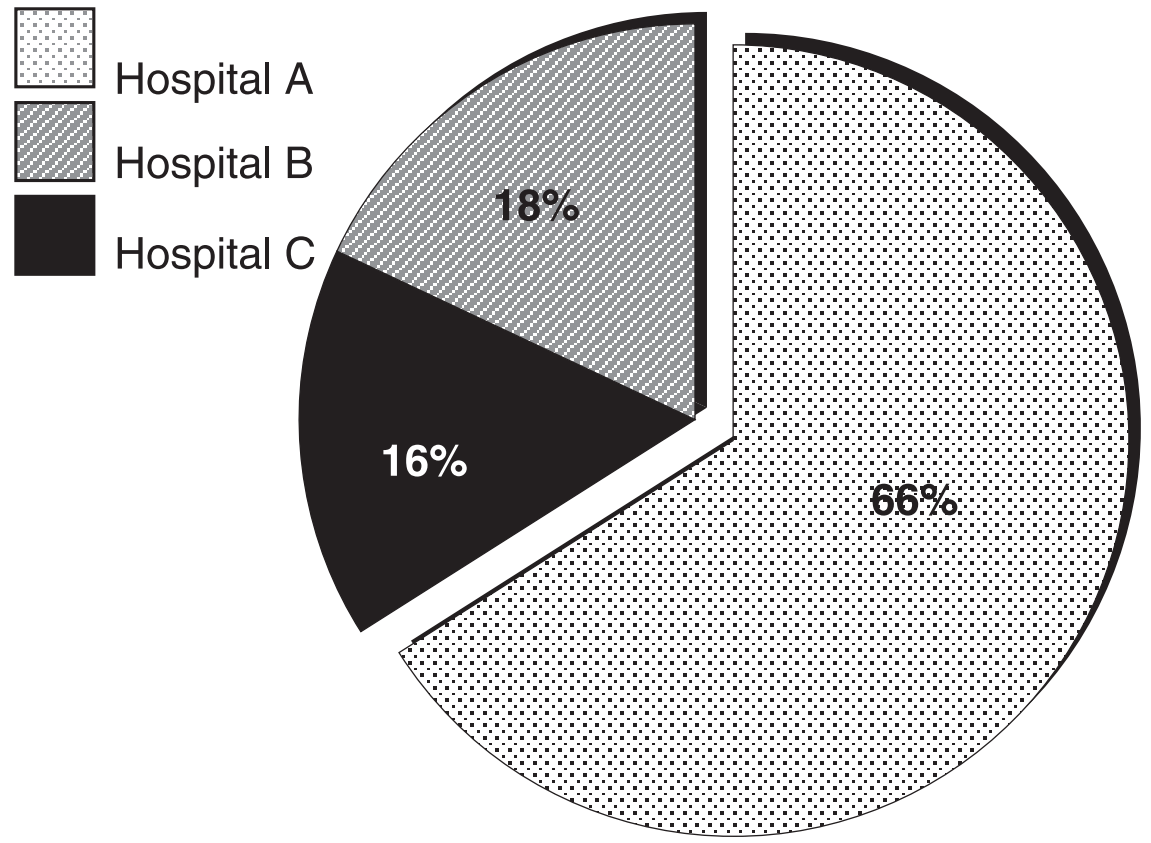

Question one stated, "What is the pain management knowledge of registered nurses practicing in acute-care hospitals in The Bahamas?" To address this question, test items from section $B$ and $C$ were combined to give a total of 30 items. However, items 19 and 26 were the same, so item 19 was used for analysis since it represented the participants' initial reaction to the question, giving an adjusted total of 29 test items. A score of one was given to each correct response and zero for incorrect or unanswered items. The total number of correct responses were divided by 29 and multiplied by 100 for a final score as a percentage value.

The mean score was $13.5(46.6 \%, s e=0.20)$ and if the participants had guessed, they could have scored $12.5(43.1 \%)$. Therefore, using a $95 \%$ probability that the participants did not guess, only 25 of 244 participants obtained a score of $18(62 \%)$ or more. Moreover, only $5.3 \%$ of the participants obtained a score of 19 or more marks. Although most of the participants $(98 \%)$ agreed that the most accurate judge of the intensity of pain is the patient him or herself, $11 \%$ thought that pain intensity should be rated by the nurse and not the patient. Most participants agreed that culture $(91 \%)$ and personality $(89 \%)$ influence a patient's pain perception and tolerance. However, no questions were answered correctly by all of the participants and question 13 relating to the equianalgesic dose of pethidine $75 \mathrm{mg}$ IM and question 15 relating to physiological response to chronic pain were almost unknown by all participants.

Additionally, more than half (54\%) of the participants could not differentiate between acute and chronic pain and more than half (56\%) of the participants thought that $25 \%$ to $50 \%$ of patients are likely to develop addiction to opioids/ narcotics when, in fact, the chance of addiction is less than $1 \%$. Fifty-six percent of the participants believed that the patient cannot sleep if experiencing severe pain and more than three quarters $(78 \%)$ of participants could not 


\section{Highest Degree Earned in Nursing}

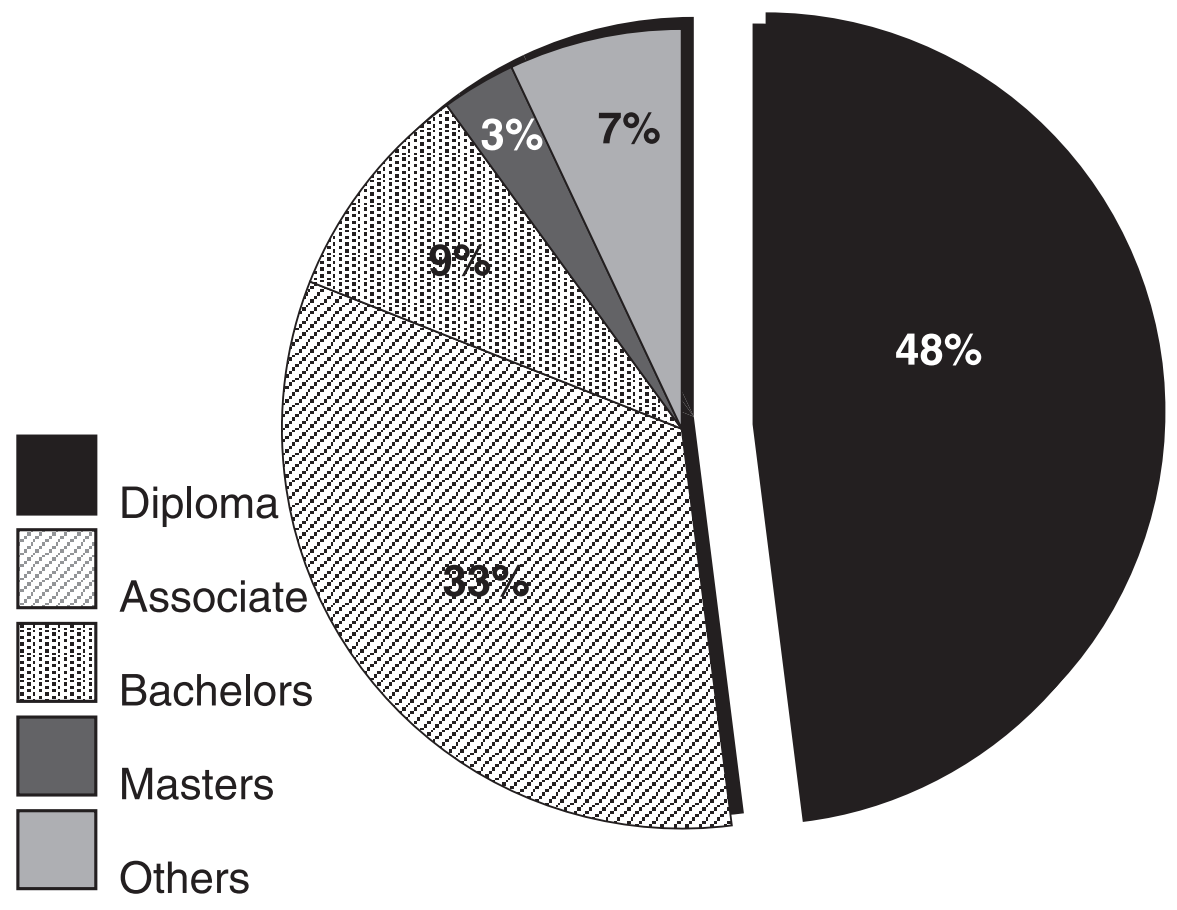

adequately assess pain because of gender biases. Seventy-eight percent of participants believed that the post-operative patient should only be given his prescribed morphine when he feels pain, and $74 \%$ of participants believed that observable changes in vital signs or behavioral changes should be present if the patient is experiencing pain. Seventy-two percent of participants believed that if a patient's pain is relieved following the administration of a placebo, this could indicate that the patient was not experiencing pain. Eighty-six percent of the participants thought that monitoring respiratory rate as opposed to sedation level is more important to prevent narcotic-induced respiratory depression. Seventy-two percent of the participants had problems differentiating pain types and about half (49\%) of the participants could not identify heroin and codeine as narcotics.

Further analyses suggest that the male participants performed better than the female participants in this study. Five of six questions where male and female participants differed most are questions that more than $68 \%$ of the participants responded to incorrectly (Table 3). The male participants never did worse than expected (guessing) for these questions; however, the female participants only did better than expected (guessing) once. Participants who showed knowledge of pain management seem to have three traits in common: $73 \%$ were between 31 and 40 years of age, $58 \%$ had a diploma as their highest educational level, and $75 \%$ worked at Hospital C.

Table 3. Percentage of male and female participants giving the correct answers to questions answered incorrectly by most participants of the study 


\begin{tabular}{|c|c|c|c|c|c|}
\hline \multirow{7}{*}{ 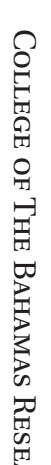 } & Question & Male & Female & $\begin{array}{c}P \text { value } \\
\text { if guessed }\end{array}$ & Expected percentage \\
\hline & 2 & 63 & 30 & 0.01 & 33 \\
\hline & 4 & 63 & 79 & $<0.001$ & 33 \\
\hline & 18 & 50 & 25 & 0.04 & 50 \\
\hline & 20 & 56 & 25 & 0.02 & 50 \\
\hline & 24 & 88 & 60 & 0.03 & 50 \\
\hline & 25 & 44 & 10 & $<0.001$ & 50 \\
\hline
\end{tabular}

Regarding the responses to question two, there is a significant difference in pain management knowledge level of registered nurses practicing in acutecare hospitals in The Bahamas among hospitals $(p<0.001)$. Participants at Hospital C had a mean score of $15.4(53 \%, s e=0.52)$ compared to means of 13.1 $(45 \%, s e=0.22)$ and $12.7(44 \%, s e=0.50)$ at Hospital A and B, respectively. Moreover the scores from the public hospitals were significantly less $(p<0.001)$ than those from the private hospital. The passing rates at the three hospitals were $2 \%$ at Hospital A, 3\% at Hospital B, and $20 \%$ at Hospital C. A regression model suggested that hospital had a significant influence on the participants' scores. Hospital C's mean scores were consistently significantly higher $(p<0.001)$ than the others, even when subsets of the data were simultaneously "balanced" for gender, degree, and years of clinical experience (Table 4). Additionally, nurses working in intensive care units significantly $(p<0.02)$ out scored (52\%) nurses working in surgical (47\%), medical (43\%) and emergency areas $(43 \%)$, respectively.

Table 4. Regression coefficients of factors linked to pain management scores

\begin{tabular}{ccc} 
Term & Coefficient & Se \\
\hline Constant & 10.47 & 1.059 \\
\hline Hospital & 0.65 & 0.295 \\
\hline Degree & 1.34 & 0.667 \\
\hline Gender & 1.94 & 0.910
\end{tabular}

Interestingly, hospital had an important effect on the participants' responses. There were clearly different responses to questions $1,4,10,20$, and 24 given by the participants at the three hospitals (Table 5). Except for questions 1 and 4, a higher percentage of participants at Hospital $\mathrm{C}$ responded correctly. Although most participants responded incorrectly to question 20 relating to the administration of a placebo and the relief of pain, participants at Hospitals A and B displayed gross lack of knowledge of this topic.

Table 5. Percentage of participants who got five questions right at the three hospitals 


\begin{tabular}{ccccc}
\hline 1 & 34 & 84 & 82 & 33 \\
\hline 4 & 66 & 84 & 82 & 33 \\
\hline 10 & 39 & 32 & 69 & 33 \\
\hline 20 & 24 & 18 & 53 & 50 \\
\hline 24 & 59 & 55 & 84 & 50
\end{tabular}

Regarding the relationship between registered nurses' practicing in acute-care hospitals in The Bahamas highest educational level and knowledge of pain management, the data supported the belief that the higher the educational level of the participants, the greater their ability to manage pain. While only participants with a master's degree had an average score that was significantly higher than the associate degree participants $(p=0.029)$, the mean scores of the groups increased as their educational level increased; Associate Degree in Nursing (44\%), Diploma (47\%), Bachelor of Science in Nursing (49\%), and Master of Science in Nursing (53\%), respectively.

In response to the last question aimed to determine the relationship between years of clinical experience and knowledge of pain management of registered nurses practicing in acute-care hospitals in The Bahamas, the findings suggested that length of experience had an effect on the participants' scores $(p=0.065)$. Nurses with less than five years' experience got a lower mean score $(12.9, s e=0.44)$ than those with more than five years of clinical experience $(14.0, s e=0.27)$.

\section{Discussion}

Congruent with other studies (Kubecka et al., 1996), findings from this study suggest that nurses in general have a good knowledge base concerning the patient as authority on their own pain, the usefulness of pain-rating scales to evaluate patients' pain, and the influence of culture and personality on patients' pain. However, with only 25 of 244 participants obtaining a score of $18(62 \%)$ or more, findings suggest that nurses lack pain management knowledge concerning physical and behavioural indicators of pain, pain assessment principles, identification of opioids/narcotics, and equianalgesia. Additionally, findings suggest that nurses practicing in acute-care hospitals in The Bahamas believe many myths concerning pain assessment and management practices.

Contrary to the results of other studies (McCaffery et al., 1990; Kubecka et al., 1996), the findings of this study suggested that the nurses' scores increased as their educational level increased. However, the only significant increase in scores noted was between nurses with associate degrees and nurses with master's degrees. As a result, it may be fair to suggest that nurses should be encouraged to seek higher education to increase their knowledge base and thus increase their ability to assess and manage patients' pain adequately.

Similarly, findings suggested that nurses with greater clinical experience scored significantly higher than their counterparts with fewer than 5 years of clinical experience. This finding may explain why nurses with a diploma as 
their highest educational level scored higher than nurses with an associate degree as their highest educational level since the diploma program was phased out over a decade ago. Additionally, it can be concluded that schools of nursing are not adequately preparing nursing students to assess and manage patients' pain since less experienced nurses showed decreased pain management knowledge. Similarly, it can also be concluded that hospitals are not providing adequate orientation and preceptorship programs, and in-service education opportunities for new nursing graduates.

Findings suggested that male nurses are more knowledgeable about pain management than their female counterparts. This may be due to the fact that most of the male participants worked at Hospital C, which scored highest in the survey. Since Hospital C is a private hospital, it might have policies and guidelines in place for pain management and greater expectation for adherence to such policies and guidelines from registered nurses. Additionally, some of the questions answered correctly by male participants and incorrectly by most female participants were related to gender effects and pain tolerance (i.e., 2 \& 4), which might have been misjudged by female nurses due to their misunderstanding pain and the male gender. However, male participants probably do not hold such myths since they know how they experience pain.

\section{Limitations of Study}

One of the limitations of this study was the way data was collected. Nurses were given the questionnaires while on duty and the questionnaires were collected during the same shift they were distributed. This might have resulted in some nurses feeling anxious and pressured to complete the questionnaires. Plus, some nurses might have been busy during their shift and that may have affected their ability to concentrate on the survey.

Since ethnicity was not addressed in the survey, there is no way of knowing how foreign-trained nurses might have affected the hospital scores obtained, especially since most foreign-trained nurses practiced in Hospital C (hospital with highest scores). Knowing the ethnicity of the nurses and their previous areas of practice before coming to The Bahamas would have shed some light on their pain management knowledge level compared to that of Bahamiantrained nurses.

\section{Nursing Implications}

As the largest group of healthcare professionals, nurses have a pivotal role to play in ensuring adequate pain assessment and management of all clients of healthcare. Nurses' responsibilities include ensuring that patients' pain is adequately assessed and managed, providing pain assessment and management information for practicing nurses and student nurses, ensuring that practicing nurses and other healthcare providers adhere to written pain-management and assessment guidelines, and ensuring that nurses engage in research initiatives to enhance adequate pain-management practices and improve the quality of life for patients experiencing pain.

The general lack of knowledge of pain management among registered nurses practicing in acute-care hospitals in The Bahamas suggests an emergent need for the following: 
(a) The inclusion of pain assessment and management credited courses in nursing curricula.

(b) Structured orientation and preceptorship programs to provide pain management information to new nursing graduates and new staff nurses in all healthcare settings.

(c) A standing committee made up of multidisciplinary teams (nurses, physicians, pharmacists, respiratory therapists, physiotherapists, dietitians) in every healthcare institution, particularly in the acutecare setting, to develop pain assessment and management protocols and procedures for all healthcare providers to follow.

(d) Willingness of healthcare institutions to sponsor interested nurses and other healthcare providers to obtain training and certification in pain assessment and management practice, to ensure that international standardized pain management guidelines tailored to meet the painmanagement needs of The Bahamas are followed within healthcare institutions.

(e) Ongoing workshops, conferences, and in-service educational opportunities offered by the institutions (collaboratively and/or singularly) to address areas of pain management such as pain assessment and documentation, including nursing care plans, standards of care, and clinical pathways; pharmacological and non-pharmacological management, including equianalgesia and addiction, dispelling myths, involving the patient, family, and/or significant other(s) in pain management practices; and working collaboratively to ensure adequate pain management to improve the quality of life for patients and to enhance customer satisfaction.

(f) Encouraging, promoting, and conducting scientific research initiatives to add to global, regional, and local knowledge about pain management and to ensure evidence-based practice. Topics for future research efforts can include: (a) Patients' rating of their own pain compared to nurses rating of patients' pain; (b) Determination of the most effective pharmacological and non-pharmacological measures to relieve pain; (c) Assessing pain-management knowledge of student nurses; (d) The effects of home remedy on pain-management in the hospitalized patients; and (e) Patients' myths about pain-management.

\section{Conclusion}

Hospitalized patients experience and complain more about pain than any other symptoms, and, since nurses are with patients more than any other healthcare provider, they witness patients' experience with pain and therefore, should know how to adequately assess and manage patients' pain. It would behoove nursing schools and hospital administrators and nurse managers to ensure that nurses are adequately prepared and equipped to perform their role in pain assessment and management practices: by ensuring that pain management courses are included in nursing curricula, new nursing graduates and staff nurses receive adequate orientation and preceptorship, and continuing education about pain management is offered to healthcare providers. Additionally, all healthcare providers should be mandated to adhere to institutional policies and practice based on established pain assessment and management protocols and procedures guidelines. It is important to note that patients whose pain is assessed and managed adequately will experience a higher quality of life and will be more satisfied with their stay in hospital. 


\section{References}

Ben David, Y., \& Musgrave, C. F. (1996). Pain assessment: A pilot in an Israeli bone marrow transplant unit. Cancer Nursing, 19(2), 93-97.

Bowman, J. M. (1994). Perception of surgical pain by nurses and patients. Clinical Nursing Research, 3(1), 69-76.

Calvillo, E. R., \& Flaskerud, J. H. (1993). Evaluation of the pain response by Mexican American and Anglo American women and their nurses. Journal of Advanced Nursing, 18, 451-459.

Camp, L. D., \& O'Sullivan, P. S. (1987). Comparison of medical, surgical, and oncology patients' descriptions of pain and nurses' documentation of pain assessments. Journal of Advanced Nursing, 12, 593-598.

Carper, B. (1978). Fundamental patterns of knowing in nursing. Advances in Nursing Science, 1(1), 13-23.

Chambers, C. T., Cassidy, K. L., McGrath, P. J., Gilbert, C. A., \& Craig, K. D. (1996). Child facial coding system (Revised). Retrieved May 27, 2002, from http://www.dal.ca/ pedpain/cfcs.cfcstitl.html.

Dalton, J. A. (1989). Nurses' perceptions of their pain assessment skills, pain management practices, and attitudes toward pain. Oncology Nursing Forum, 16(2), 225-231.

Dufault, M. A., Bielecki, C., Collins, E., \& Willey, C. (1995). Changing nurses' assessment practice: A collaborative research utilization approach. Journal of Advanced Nursing, 21, 634-645.

Ferrell, B. R., McCaffery, M., \& Grant, M. (1991). Clincal decision making and pain. Cancer Nursing, 14(6), 289-297.

Ferrell, B. R., McCaffery, M., \& Ropchan, R. (1992). Pain management as a clinical challenge for nursing administration. Nursing Outlook, 40(6), 263268.

Gujol, M. C. (1994). A survey of pain assessment and management practices among critical care nurses. American Journal of Critical Care, 3(2), 123128.

Harrison, A. ( 1991). Assessing patients' pain: Identifying reason for error. Journal of Advanced Nursing, 16, 1018-1025.

Herr, K. A., \& Mobily, P. R. (1993). Comparison of selected pain assessment tools for use with the elderly. Applied Nursing Research, 6(1), 39-46.

Ignatavicius, D. D., Workman, M. L., \& Mishler, M. A. (1999). Medical surgical nursing across the health care continuum. (3rd ed.). Philadelphia: Saunders.

Jacobs-Kramer, M. K., \& Chinn, P. L. (1988). Perspectives on knowing: A model of nursing knowledge. Scholarly Inquiry for Nursing Practice, 2(2), 129144.

Kubecka, K. E., Simon, J. M., \& Boettcher, J. H. (1996). Pain management knowledge of hospital-based nurses in a rural Appalachian area. Journal of Advanced Nursing, 23, 861-867.

McCaffery, M., \& Ferrell, B. R. (1992). How vital are vital signs? Nursing, 22(1), 42-46.

McCaffery, M., Ferrell, B., O’Neil-Page, E., \& Lester, M. (1990). Nurses' knowledge of opioid analgesic drugs and psychological dependence. Cancer Nursing, 13(1), 21-27.

McDonald, D., McNulty, J., Erickson, K., \&Weiskopf, C. (2000). Communicating pain and pain management needs after surgery. Applied Nursing Research, 13, 70-75.

Nash, R., Edwards, H., \& Nebauer, M. (1993). Effect of attitudes, subjective 
norms and perceived control in nurses' intention to assess patients' pain. Journal of Advanced Nursing, 18, 941-947.

Phillips, D. M. (2000). JCAHO pain management standards are unveiled. [Electronic version]. Journal of the American Medical Association, 248(4), 428-429.

Rutledge, D. N., \& Donaldson, N. E. (1998a). Pain assessment and documentation, Part 1: Overview and application in adults. Online Journal of Clinical Innovations, 1(5), 1-37.

Rutledge, D. N., \& Donaldson, N. E. (1998b). Pain assessment and documentation, Part 2: Special populations of adults. Online Journal of Clinical Innovations, 1(6), 1-29.

Simons, W., \& Malabar, R. (1995). Assessing pain in elderly patients who cannot respond verbally. Journal of Advanced Nursing, 22, 663-669.

Wells, N. (1984). Responses to acute pain and the nursing implications. Journal of Advanced Nursing, 9, 51-58.

White, C. L. (1999). Changing pain management practice and impacting on patient outcomes. Clinical Nurse Specialist, 13(4), 166-72.

Willson, H. (2000). Factors affecting the administration of analgesia in patients following repair of fractured hip. Journal of Advanced Nursing, 31, 11451154.

Yates, P., Dewar, A., Edwards, H., Fentiman, B., Najman, J., Nash, R., et al. (1998). The prevalence and perception of pain amongst hospital inpatients. Journal of Clinical Nursing, 7, 521-530.

Shane Neely-Smith is a lecturer in the School of Nursing \& Allied Health Professions at The College of The Bahamas. She is presently on study leave, pursuing a Ph.D. in Nursing at Barry University.

Maggie Turner is the Chairperson and Senior Lecturer in the School of Nursing at The College of The Bahamas.

Zorene A. Curry is a Senior Lecturer in the School of Nursing at The College of The Bahamas where she heads the Department of Nursing and Allied Health. She is working towards a Ph.D. in Nursing.

Theresa E.Moxey-Adderley is a Lecturer in the School of Nursing \& Allied Health Professions at The College of The Bahamas.

Constance J. Wilson is the Coordinator of the Bachelor in Nursing programme, and Lecturer in the School of Nursing and Allied Health Professions at The College of The Bahaamas.

Sandra G. Smith is an Advanced Nursing Administrator engaged in Research with the Ministry of Health, Nassau, Bahamas. 